\title{
LDPC Coded Modulation with Probabilistic Shaping for Optical Fiber Systems
}

\author{
Tobias Fehenberger $^{(1)}$, Georg Böcherer ${ }^{(1)}$, Alex Alvarado ${ }^{(2)}$ and Norbert Hanik $^{(1)}$ \\ (1) Institute for Communications Engineering, Technische Universität München, 80333 Munich, Germany \\ ${ }^{(2)}$ Optical Networks Group, University College London (UCL), London, WC1E 7JE, UK \\ tobias.fehenberger@tum.de
}

\begin{abstract}
An LDPC coded modulation scheme with probabilistic shaping, optimized interleavers and noniterative demapping is proposed. Full-field simulations show an increase in transmission distance by $8 \%$ compared to uniformly distributed input.
\end{abstract}

OCIS codes: 060.4080 Modulation, 060.4510 Optical communications.

\section{Introduction}

In the pursuit of higher data rates in optical communications, high-order modulation formats are an established technique to increase spectral efficiencies. As the optical channel is effectively power-limited by the nonlinearities, optimizing the signaling is necessary in order to further improve the spectral efficiency without increasing the launch power. Probabilistic shaping and geometric shaping are two possibilities to achieve this. The former uses constellations with nonuniform distributions on a regular grid while the latter uses nonequidistant spacings of equiprobable symbols. The main implementation advantage of probabilistic shaping over geometric shaping is that it does not require modifications of the digital-to-analog converters and the optical signal processing algorithms.

Probabilistic shaping has been proposed for optical transmission, e.g. in [1-3]. In [1], a multi-level coded modulation scheme with trellis shaping and hard-decision decoding is found to operate close to a capacity estimate. Probabilistic shaping via shell-mapping improving a 4D coded modulation scheme is presented in [2]. In [3], probabilistic shaping is implemented for turbo codes by a many-to-one mapping. This type of mapping, however, requires information exchange between the demapper and the decoder at the receiver, known as iterative demapping.

In this paper, we adapt the probabilistic shaping scheme introduced in [4] to a multi-span wavelength-division multiplexing (WDM) optical fiber system with quadrature amplitude modulation (QAM). Off-the-shelf binary encoders and decoders are used and no iterative demapping is required. Using bit-interleaved coded modulation and a lowdensity parity-check (LDPC) code, the presented scheme increases the transmission distance by $8 \%$. To the best of our knowledge, this is the first demonstration of such gains based on probabilistic shaping in optical communications.

\section{Mutual Information Analysis}

\subsection{Optical Transmission Setup}

The setup of the optical coded modulation system is shown in Fig. 1. The QAM symbols are either uniformly distributed or shaped as explained in Sec. 2.2. We consider a dual-polarization multi-span WDM system with 15 copropagating channels. The baud rate per channel is 28 GBaud, the pulses are root-raised-cosine (RRC) shaped with $5 \%$ roll-off, the WDM spacing is $30 \mathrm{GHz}$. We simulate $2^{16}$ symbols per polarization. The fiber is a standard singlemode fiber (SSMF) with $\alpha=0.2 \mathrm{~dB} / \mathrm{km}, \gamma=1.3(\mathrm{~W} \cdot \mathrm{km})^{-1}$ and $D=17 \mathrm{ps} / \mathrm{nm} / \mathrm{km}$. Each span of length $100 \mathrm{~km}$ is followed

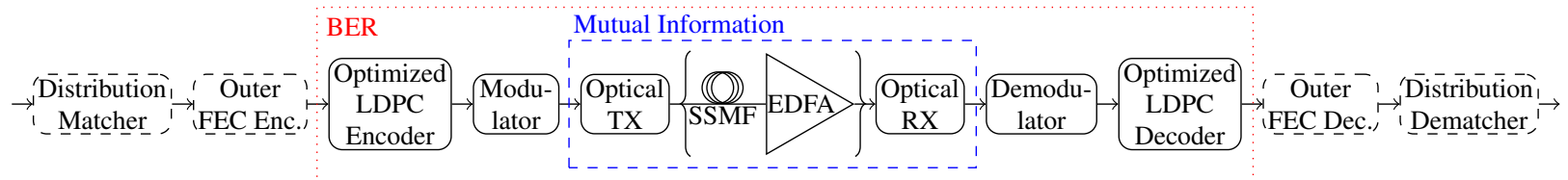

Fig. 1: Block diagram of the coded modulation system with probabilistic shaping. Each span consists of $100 \mathrm{~km}$ SSMF and an EDFA. 


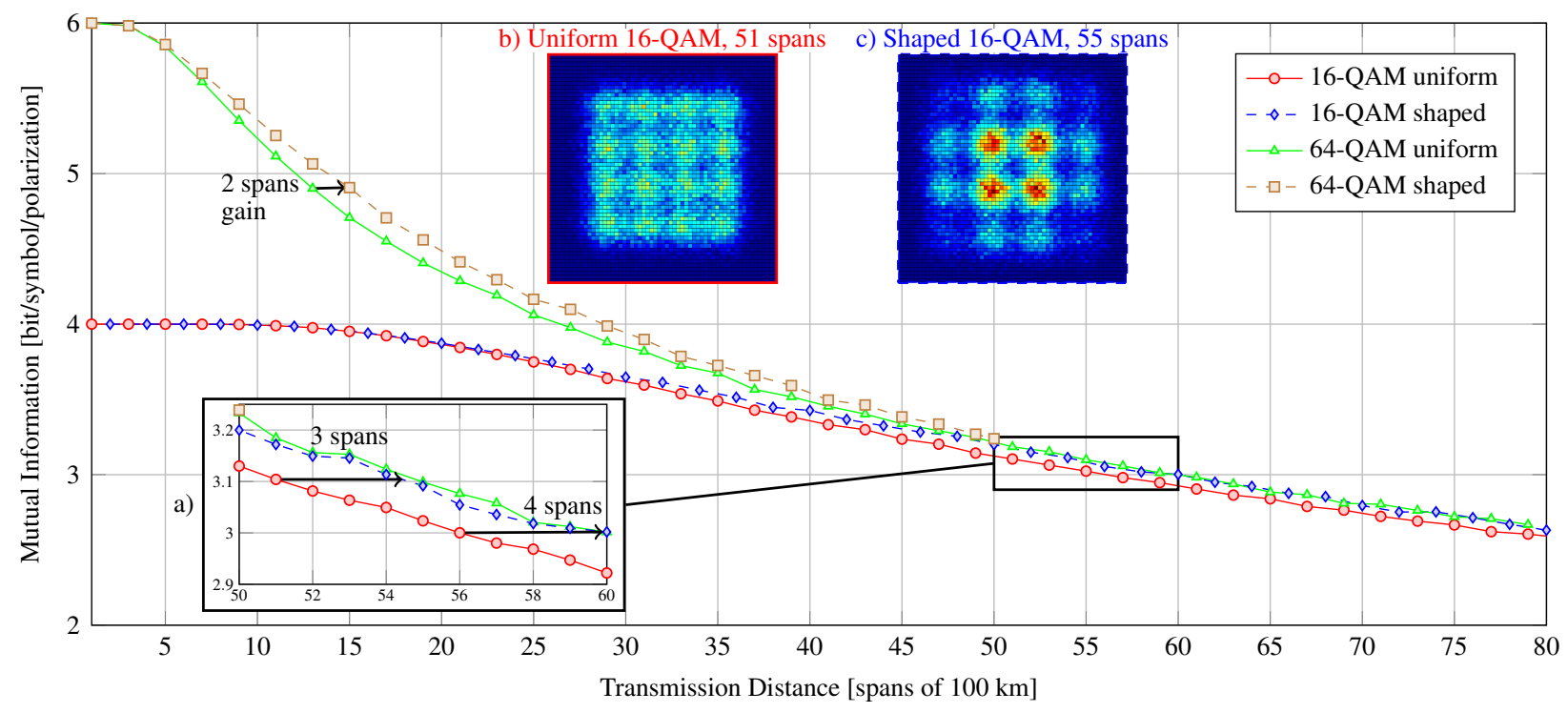

Fig. 2: MI versus distance. a): Zoom from 50 to 60 spans. b) and c): Distributions of the received symbols for uniform and shaped input, resp.

by an Erbium-doped fiber amplifier (EDFA) with a noise figure of $4 \mathrm{~dB}$. Signal propagation is simulated using the split-step Fourier method with 32 samples per symbol and a step size of $100 \mathrm{~m}$. At the receiver, the center WDM channel is bandpass-filtered and converted into the digital domain with a coherent receiver. Chromatic dispersion is compensated digitally and an RRC filter is applied again. Laser phase noise and polarization mode dispersion are not included in the simulations as perfect compensation is assumed. We compute nonparametric estimates of the mutual information (MI) between input and output symbols without considering memory (see the dashed blue box in Fig. 1). In particular, Gaussian statistics are not assumed a priori for MI estimation.

\subsection{Optimized Input Distribution}

We probabilistically shape 16-QAM and 64-QAM by assigning larger probabilities to the points with lower energy. At the same power, this increases the Euclidean distance between constellation points compared to uniform input.

To find a suitable distribution for the shaped input, we first resort to the Gaussian noise (GN) model [5] and calculate the signal-to-noise ratio (SNR) for every transmission distance. We then follow [6] and jointly optimize the MaxwellBoltzmann distribution of the input symbols and the constellation scaling such that the MI is maximized for the additive white Gaussian noise (AWGN) channel under the power constraint imposed by the SNR. The obtained distribution is used for the shaped input of the optical fiber simulations.

Insets b) and c) of Fig. 2 illustrate the effect of uniform and shaped input on the received symbol distributions, respectively, with hotter colors representing a larger number of occurrences. The innermost points of the shaped input are sent more often and the Euclidean distance between constellation points is $18 \%$ larger than for uniform input.

\subsection{Mutual Information Results}

MI versus transmission distance is shown in Fig. 2 for 16-QAM and 64-QAM, both with uniform and shaped input. For each overall transmission distance of 1 to 80 spans, we use an optimized shaped input and determine a candidate for the optimal launch power from the GN model. By testing different launch powers in our simulations, we found that the optimum is within $0.2 \mathrm{~dB}$ of $-1.6 \mathrm{dBm}$ for all simulated modulations and distances.

For small distances, shaped and uniform QAM achieve similar MI because the impact of distortions is small in this region and the shaped distribution is close to the uniform distribution. Using shaped 64-QAM at 15 spans, however, gives 2 additional spans compared to uniform input, which is an increase of $15 \%$. For longer distances, the improvement over uniform 64-QAM disappears, and thus, we did not simulate shaped 64-QAM for more than 50 spans. Once the signal propagates further and distortions accumulate, we observe that shaped 16-QAM performs better than uniform 16-QAM. From 50 spans onwards, the MI curves of shaped 16-QAM and uniform 64-QAM coincide. Inset a) of Fig. 2 shows that shaped 16-QAM increases the transmission distance by 3 to 4 spans compared to uniform 16-QAM. 


\section{BER Analysis}

To verify our MI results, we implement an LDPC code as inner forward error correction (FEC) and compare the BERs of uniform and shaped 16-QAM (see the dotted red box in Fig. 1). We operate at an information rate of 3 bits per symbol per polarization, without considering the coding overhead $(\mathrm{OH})$ of the outer FEC. The LDPC codes of our coded modulation scheme are from the DVB-S2 standard and have a block length of 64800 bits.

\subsection{Implementation}

A total of four bits map to one 16-QAM symbol. For uniform input and a target information rate of 3 bits per symbol, we use 3 bits for data and one bit for redundancy. The rate 3/4 LDPC code is used to achieve this. For our shaped input, we effectively map 3 uniformly distributed data bits to 3.2 shaped bits. We use the remaining $4-3.2=0.8$ bits per QAM symbol for redundancy by encoding the shaped data with a rate 3.2/4 $=4 / 5$ LDPC code. Hence, the overall redundancy of one bit is successively added in two steps, i.e., by probabilistic shaping and by LDPC encoding. The mapping from uniformly distributed data bits to shaped bits is done by a distribution matcher [7] as shown in Fig. 1. In our simulations, we emulated the distribution matcher output by directly generating the shaped bits.

We optimize the LDPC codes by wrapping the encoder with two interleavers and the decoder with the respective inverse interleavers. The interleavers optimize the mapping of the coded bits to the QAM symbols and ensure that the distribution imposed on the data is preserved at the modulator output $[4, \mathrm{Sec}$. V-D].

A staircase code with $6.7 \% \mathrm{OH}[8]$ is assumed as outer code. It must be placed between the distribution matcher and the inner FEC to avoid error propagation. A systematic encoder must be used to preserve the statistics generated by the distribution matcher.

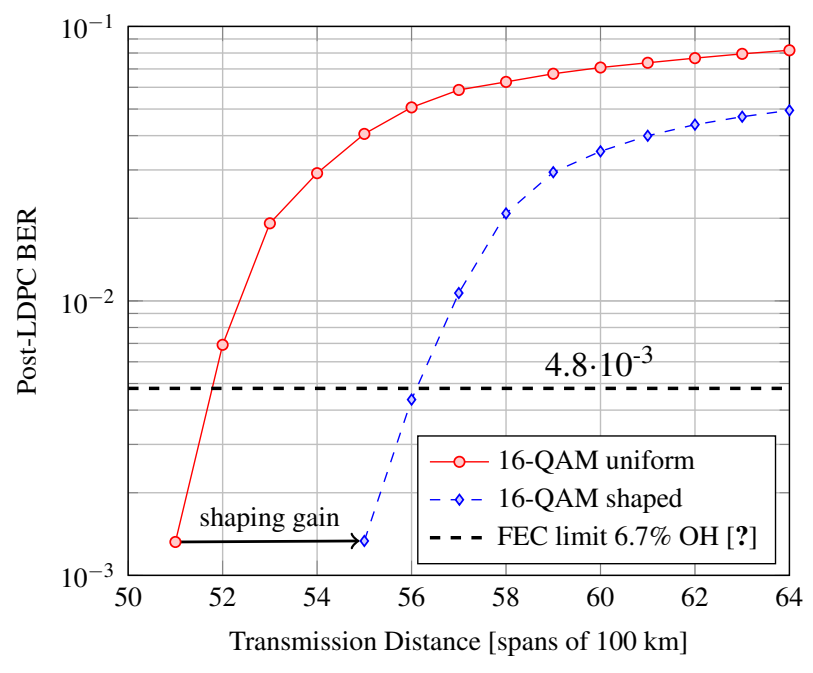

\subsection{BER Results}

Fig. 3: BER after LDPC decoding at 3 bits per symbol information rate.

In Fig. 3, the plot of BER versus distance shows that the shaping gain predicted by the MI in Fig. 2 translates into an improvement in BER. At a BER of $1.3 \cdot 10^{-3}$, we observe a shaping gain of 4 spans compared to uniform 16-QAM. This increase in transmission distance by $8 \%$ is in excellent agreement with the prediction of the MI curves in Fig. 2.

\section{Conclusions}

We have presented an LDPC coded modulation scheme with probabilistic shaping. Compared to previously suggested shaping schemes, the implementation has lower complexity because no iterative demapping is required at the receiver. We show in optical fiber simulations that 16-QAM and 64-QAM with shaped input allow for an increase in transmission distance by $8 \%$ and $15 \%$, respectively, compared to uniformly distributed input. The results are promising and further gains are expected by optimizing the input distribution for the nonlinear optical channel. Also, four-dimensional constellations that are more robust against nonlinear distortions will be considered in future work.

\section{References}

1. B.P. Smith and F. R. Kschischang, "A pragmatic coded modulation scheme for high-spectral-efficiency fiber-optic communications," J. Lightw. Technol. 30(13), pp. 2047-2053, Jul. 2012.

2. L. Beygi et al., "Rate-adaptive coded modulation for fiber-optic communications," J. Lightw. Technol. 32(2), pp. 333-343, Jan. 2014.

3. M.P. Yankov et al., "Constellation shaping for fiber-optic channels with QAM and high spectral efficiency," Photon. Technol. Lett. 26(23), pp. 2407-2410, Dec. 2014.

4. G. Böcherer, "Probabilistic signal shaping for bit-metric decoding," in Proc. Int. Symp. Inf. Theory (ISIT), pp. 431-435, Jul. 2014.

5. P. Poggiolini et al., "The GN-model of fiber non-linear propagation and its applications," J. Lightw. Technol. 32(4), pp. 694-721, Feb. 2014.

6. F.R. Kschischang and S.Pasupathy, "Optimal nonuniform signaling for Gaussian channels," Trans. Inf. Theory 39(3), pp. 913-929, May 1993.

7. S. Baur and G. Böcherer, "Arithmetic Distribution Matching," [Online]. Available: http: //arxiv. org/abs/1408.3931, Aug. 2014.

8. B.P. Smith et al., "Staircase codes: FEC for 100 Gb/s OTN," J. Lightw. Technol. 30(1), pp. 110-117, Jan. 2012. 